\title{
Progresos de radioquimioterapia en cáncer de pulmón no microcítico
}

\author{
R. Morera, A. Montero, A. Hervás, I. Rodríguez, S. Sancho, S. Córdoba, J. Corona, A. Ramos
}

\section{Introducción}

El cáncer de pulmón no microcítico (CPNM) estadio III, definido como un tumor localmente avanzado, limitado a tórax y sin evidencia de metástasis a distancia, representa el $35 \%$ del total de neoplasias pulmonares. En estos pacientes, el tratamiento con cirugía o radioterapia obtiene unos pobres resultados, con una supervivencia a 5 años inferior al $10 \%$. Esta baja tasa de curación se debe a la imposibilidad de estos tratamientos para eliminar completamente el tumor primario y erradicar las micrometástasis presentes en el momento del diagnóstico. Con el objetivo de mejorar estos resultados se han investigado nuevas estrategias terapéuticas basadas en:

A) Combinación de QT y RT de forma recurrencial o concurrente.

B) Tratamiento preoperatorio con QT o QT-RT en determinados subgrupos de pacientes con tumores estadio III potencialmente resecables.

C) Intensificación de la RT mediante la utilización de nuevos esquemas de tratamiento (esquemas de hiperfraccionamiento, RTE conformacinal 3D, IMRT).

\section{QT-RT preoperatoria en estadio III de CPNM potencialmente resecable}

En los estudios fase II y III en pacientes con CPNM estadio III, los esquemas de QT neoadyuvante, basados en combinaciones con cisplatino, han obtenido un elevado porcentaje de respuestas radiológicas. Sin embargo, en menos del 10\% de pacientes se objetiva una respuesta patológica completa. Con el objetivo de mejorar estos resultados, diversos estudios han analizado la actividad de la combinación de QT y RT, con una dosis de 45 a 50 Gy en un fraccionamiento estándar de 1,8-2 Gy. La combinación de estos tratamientos ten-

Servico de Oncología Radioterápica

Hospital Ramón y Cajal

Madrid dría la ventaja de aprovechar el potencial radiosensibilizador demostrado por los citostáticos activos en el CPNM, que permitiría obtener un mejor control local del tumor. En este sentido, los estudios de quimiorradioterapia preoperatorio han obtenido entre un $20 \%$ y un $30 \%$ de respuestas completas patológicas, porcentaje significativamente superior al demostrado por los esquemas de QT neoadyuvante. En contrapartida, la combinación de ambos tratamientos aumenta notablemente la toxicidad aguda y, posiblemente, también las complicaciones postoperatorias.

En los estudios de quimiorradioterapia preoperatorio en pacientes con estadio III, se ha observado que determinados subgrupos de pacientes con tumores estadio IIIB podrían beneficiarse del tratamiento combinado, según los criterios de Eberhardt' ${ }^{1}$.

Los estudios de QT y RT preoperatorias han obtenido una mediana de supervivencia de 13 a 25 meses y una supervivencia a 5 años del 18 al $37 \%$.

En un estudio del CALGB (Cancer and Leucemia Group B), publicado por Strauss et al en 1992, 41 pacientes con CPNM estadio IIIA (80\% de ellos N2), fueron tratados con un esquema preoperatorio de dos ciclos de cisplatino/fluoruracilo/vinblastina y RT concomitante (dosis de $30 \mathrm{~Gy}$ ) con un ciclo adicional de QT y RT (30 Gy) en el postoperatorio. La tasa de resecabilidad en este estudio fue del $77 \%$, con un $23 \%$ de respuestas patológicas completas. La mediana de supervivencia y la supervivencia a un año fue de 15,5 meses y del $58 \%$ respectivamente. La principal limitación del estudio fue la elevada toxicidad, con un 15\% de muertes relacionadas con el tratamiento?

En el estudio del Southwest Oncology Group (SWOG$8805)^{3}, 126$ pacientes con CPNM estadio III (75 pacientes con estadio IIIAN2 y 51 pacientes con estadio IIIB) fueron tratados con cisplatino/etopósido (2 ciclos) y RT concomitante (45 Gy). Este esquema ha demostrado una elevada eficacia, con un $58 \%$ de respuestas patológicas $(21 \%$ de respuestas patológicas completas y un $37 \%$ de casos sólo con focos microscópicos de tumor). La supervivencia global a 3 y 6 años fue del $26 \%$ y el $20 \%$, respectivamente. La supervivencia fue similar en los pacientes con estadio IIIAN2 y IIIB con una supervivencia mediana y a tres años de 13 y 17 meses $y$ del $27 \%$ y el $24 \%$, respectivamente $(p=0,81)$. Un hallazgo relevante de este estudio fue la ausencia de una correlación fidedigna entre la respuesta radiológica (valorada mediante la TAC) y la respuesta patológica puesto que en doce de los 
26 pacientes valorados por TAC como enfermedad estable se objetivó una respuesta patológica significativa. Es de destacar que los pacientes IIIB estadificados como T4 NO- 1 obtuvieron una supervivencia del $50 \%$ a los seis años con la resección quirúrgica después de presentar una estabilización de la enfermedad. Este estudio también muestra el impacto del downstaging desde N2/3 a N0 con el tratamiento de inducción sobre la supervivencia: $33 \%$ vs $11 \%$ a los seis años $(p=0,002)^{4}$.

En un estudio publicado recientemente por Grunenwald et al. se ha analizado la actividad de la quimiorradioterapia preoperatoria exclusivamente en pacientes con CPNM estadio IIIB. Un total de 40 pacientes (21 con tumores T4 y 19 pacientes con afectación N3), diagnosticados mediante toracotomía o mediastinoscopia, fueron tratados con un esquema de QT con 2 ciclos de cisplatino/fluoruracilo/vinblastina y RT concomitante (42 Gy en 2 ciclos separados por 10 días). La resección quirúrgica completa fue posible en el $60 \%$ de pacientes. En un $30 \%$ de pacientes con metástasis linfáticas mediastínicas (cN2-3) se objetivó la desaparición del tumor a este nivel después del tratamiento de inducción (pNO1); en estos pacientes la supervivencia a cinco años fue del $42 \%$, mientras que en los pacientes con persistencia en la afectación mediastínica (pN2-3) , la supervivencia disminuía al $12 \%$ a los cinco años ${ }^{5}$.

Diversos estudios han valorado la actividad de nuevos esquemas de RT con hiperfraccionamiento en combinación con los esquemas clásicos de QT. En el estudio del Massachussets General Hospital ${ }^{6}, 42$ pacientes con estadio IIIA(N2) fueron tratados con dos ciclos de cisplatino, vinblastina y fluoruracilo más RT concomitante (42 Gy administrados en 2 fracciones/día de 1,5 Gy en dos ciclos de 21 Gy separados 10 días). En el postoperatorio se administró un ciclo adicional de QT concomitante con una dosis de RT de 12 a $18 \mathrm{~Gy}$. El tratamiento de inducción obtuvo un $74 \%$ de respuestas clínicas y un $67 \%$ de respuestas patológicas (downstaging). La supervivencia a cinco años fue del $37 \%$.

En un segundo estudio, 94 pacientes con CPNM estadio IIIA y IIIB fueron tratados con tres ciclos de cisplatino/etopósido seguido de un cuarto ciclo con RT hiperfraccionada concomitante (45 Gy 1,5 Gy BID). En un 53\% de pacientes se realizó una resección quirúrgica completa y en 24 pacientes (26\%) se objetivó una respuesta completa patológica. La supervivencia a cuatro años fue de $31 \%$ y $26 \%$ en los pacientes con estadio IIIA y IIIB, respectivamente?.

En el tercer estudio con RT hiperfraccionada llevado a cabo por Thomas et $a^{8}{ }^{8}, 54$ pacientes con estadio IIIA-B fueron tratados con dos ciclos de inducción con ifosfamida/carboplatino/etopósido seguido de RT (45 Gy 1,5 Gy BID) concomitante con carboplatino/vindesina. La supervivencia mediana fue de 25 y 17 meses en los estadios IIIA y IIIB, respectivamente.

Por último, añadiremos que estudios en marcha actuales tendrán que determinar si pacientes con tumores localmente avanzados se beneficiarán más de QT-RT de inducción seguida de cirugía o de QT-RT concurrente sola?

\section{QT-RT concurrente en estadio III de CPNM}

El beneficio de añadir QT a RT para estadio III de CPNM está bien establecido.
En la última década, diversos estudios aleatorizados han demostrado que en los pacientes con CPNM localmente avanzado estadio III, las combinaciones de QT basadas en cisplatino seguido de RT mejora la supervivencia respecto al tratamiento con RT sola.

En el estudio de Dillmam et al (CALGB 8430) la combinación de dos ciclos de cisplatino y vinblastina seguido de RT (60 Gy) demostró un aumento significativo de la supervivencia mediana (13,7 vs 9,5 meses) y de la supervivencia a 5 años $(17 \%$ vs $6 \%)$ respecto al tratamiento con RT sola 160 Gy) $)^{10}$.

En un estudio posterior de Le Chevalier et al se demuestra un aumento en la supervivencia mediana de dos meses y del $7 \%$ a los dos años con un esquema de QT (cisplatino/vindesina/ciclofosfamida/lomustina) seguido de RT respecto a la RT sola. Este beneficio se obtuvo a expensas de una disminución significativa en la incidencia de metástasis a distancia, del $70 \%$ al $49 \%$, en los pacientes tratados con el esquema combinado respecto a los que recibieron tratamiento con RT sola ${ }^{11}$.

En un estudio del Radiotherapy Oncology Group (RTOG 8808), se compararon los dos esquemas del estudio de Dillman con un tercer brazo de RT sola administrada en un esquema de hiperfraccionamiento. En este estudio se incluyeron pacientes con estadio IIIA y IIIB con características de buen pronóstico (índice de Karnofsky igual o superior al $70 \%$, una pérdida de peso no superior al $5 \%$ y ausencia de derrame pleural y de adenopatías supraclaviculares). En los pacientes tratados con QT y RT secuencial, la supervivencia mediana y a los dos años fue superior que en los pacientes tratados con RT estándar y con RT hiperfraccionada: 13,7 meses y $32 \%, 11,4$ meses y $11 \%, 12,2$ meses y $24 \%$, respectivamente. Sin embargo, la supervivencia a cinco años fue inferior al $10 \%$ en los tres esquemas de tratamiento $18 \%, 5 \%$ y $6 \%$, respectivamente) $)^{12}$.

Desde 1997, el beneficio de añadir QT a la RT ha sido corroborado por dos estudios prospectivos fase III. El estudio prospectivo más grande fue conducido por RTOG, ECOG y SWOG aleatorizando a 490 pacientes a recibir dos meses de cisplatino/vinblastina seguidos de RT (60 Gy) a 2 Gy por fracción, o uno de los dos brazos de RT sola. La supervivencia global fue estadísticamente superior para los pacientes que recibieron QT y RT vs los otros dos brazos del estudio. Sin embargo, el beneficio de supervivencia de la modalidad combinada de tratamiento, en este estudio fue sustancialmente menor que la vista en el estudio $\mathrm{CALGB}^{13}$. Las formas de primer fracaso mostraron menos metástasis a distancia para pacientes que recibieron QT comparada con los brazos de RT sola. Los resultados de las revisiones Cochrane coincidieron también en demostrar un beneficio en supervivencia a favor de QT (Cochrane Database of Systematic Reviews:CD002139, 2000).

Otra estrategia de tratamiento en el CPNM estadio III es la administración de RT y QT concomitante. Estos esquemas se basan en el potencial radiosensibilizador de determinados citostáticos, especialmente los derivados del platino. La administración de QT concurrente con RT, teóricamente mejora el control local por sensibilización del tumor a la radiación, mientras que simultáneamente se trata la enfermedad sistémica, aunque si bien aumenta la toxicidad local.

En el estudio aleatorizado de la EORTC, la combinación de cisplatino (semanal o diario) concomitante con RT demos- 


\section{R. Morera y cols.}

tró un aumento significativo de la supervivencia respecto a los pacientes tratados con RT sola (supervivencia a tres años del $16 \%$ y el $2 \%$, respectivamente). Este beneficio fue a expensas de un incremento en el control local del tumor en los pacientes tratados con quimiorradioterapia, especialmente con el esquema de RT y cisplatino administrado diariamente, superior a los resultados obtenidos con cisplatino semanal. La supervivencia libre de progresión local a un año fue del $59 \%, 42 \%$ y el $41 \%$ con el esquema de cisplatino diario, cisplatino semanal y RT sola, respectivamente ${ }^{14}$.

Sin embargo, en un estudio fase III posterior llevado a cabo por el CALGB y el ECOG, 283 pacientes con CPNM estadio III fueron aleatorizados después de dos ciclos de cisplatino/vinblastina a recibir tratamiento con RT sola o RT concomitante con carboplatino (dosis de $100 \mathrm{mg} / \mathrm{m}^{2} /$ semanal). La supervivencia mediana fue prácticamente idéntica en ambos esquemas (13,5 meses y 13,4 meses, respectivamente), a pesar de que el control local del tumor fue superior en los pacientes tratados con carboplatino y RT (tasa de recidivas en el campo de irradiación del $69 \%$ en los pacientes tratados con RT sola y del $59 \%$ con el esquema de quimiorradioterapia concurrente) $)^{15}$.

En el estudio de Lee et al, se analizó el papel de la RT hiperfraccionada (dosis total de 69 Gy en fracciones de 1, 2 Gy, 2 sesiones/día) concomitante con 2 ciclos de cisplatino $\left(75 \mathrm{mg} / \mathrm{m}^{2}\right.$ día 1) y etopósido oral $(100 \mathrm{mg} /$ día, días $1-14)$ en 79 pacientes con CPNM estadio IIIA y IIIB irresecables. La tasa de respuestas al tratamiento fue del $70 \%$ (36\% de respuestas completas) y la supervivencia mediana fue de 18,9 meses, superior a la obtenida en los estudios con QT y RT secuencial. La principal limitación de este esquema fue la toxicidad, puesto que el $57 \%$ de los pacientes presentaron toxicidad hematológica grado 4, el 53\% esofagitis grado 3-4, y el $25 \%$ toxicidad pulmonar grado $3-4^{16}$.

Recientemente, dos estudios aleatorizados han comparado el tratamiento con QT y RT administradas de forma secuencial o concomitante. En ambos estudios se ha demostrado un beneficio (tanto en control local como en supervivencia) con el tratamiento concomitante respecto al esquema secuencial.

En el estudio japonés de Furuse se randomizaron 320 pacientes a recibir QT-RT concurrente o secuencial. El brazo de concurrente recibió cisplatino/vindesina/mitomicina durante cinco semanas concurrente con RT en dos cursos de 28 Gy (2 Gy por fracción durante 14 días, 5 días por semana) separados por un período de descanso de 10 días. El brazo secuencial recibió la misma QT, pero la RT fue iniciada después de completar la QT, administrándose 56 Gy (2 Gy por fracción y 5 días por semana, hasta un total de 28 fracciones). La tasa de respuestas objetivas ( $87 \%$ vs $66 \%$ ), la supervivencia mediana (16,5 meses 13,3 meses) y la supervivencia a 5 años $(15,8 \%$ vs $8,9 \%)$, fue significativamente superior en los pacientes tratados con el régimen concomitante. En el análisis multivariante el esquema de tratamiento (secuencial vs concomitante) y el performance status (0-1 vs 2) fueron las únicas variables con significación pronóstica. La toxicidad hematológica (leucopenia y plaquetopenia grado 3-4) fue superior con el tratamiento concomitante, mientras que el porcentaje de esofagitis grado 3-4 fue similar en ambos esquemas de tratamiento ${ }^{17}$.

En el segundo estudio (protocolo RTOG 9410), 611 pacientes con CPNM estadio II inoperable, IIIA y IIIB fueron aleatorizados en tres esquemas de tratamiento:
1. QT y RT secuencial (cisplatino/vinblastina seguido de $60 \mathrm{~Gy})$.

2. QT y RT concomitante (cisplatino/vinblastina concurrente con $60 \mathrm{~Gy})$.

3. QT y RT en esquema de hiperfraccionamiento concomitante (cisplatino/etopósido concurrente con 69,6 Gy administrados en dos fracciones diarias)

La supervivencia mediana fue significativamente superior con el tratamiento concomitante (esquema 2) que con el tratamiento secuencial (esquema 1): 17 meses y 14,6 meses respectivamente $(p=0,038)$. Por el contrario, el tratamiento con RT hiperfraccionada y QT concomitante (esquema 3) no demostró una mejoría significativa de la supervivencia, a pesar de obtener un mejor control local del tumor, respecto al tratamiento secuencial. Respecto a la toxicidad de estos esquemas, la tasa de esofagitis grado 3-4 fue significativamente superior con los esquemas de concurrencia respecto al tratamiento secuencial ${ }^{18}$

Otros regímenes de QT han sido testados con RT concomitante, habiéndose probado ser seguros en estudios fase II, incluyendo carboplatino/paclitaxel, gemcitabine/cisplatino, paclitaxel/cisplatino y cisplatino/vinorelbine. Estos esquemas de quimiorradioterapia esperan ser testados en estudios fase III.

Los estudios aleatorizados presentados anteriormente, demuestran de forma clara el beneficio del tratamiento concomitante con QT y RT respecto al esquema secuencial. A partir de estos resultados, en los últimos años se ha iniciado una nueva fuente de debate para conocer si la adición de QT de inducción o de consolidación a la quimiorradioterapia concurrente aporta algún beneficio. El objetivo de estos esquemas de tratamiento sería mejorar el control sistémico del tumor mediante la administración de dosis plenas de QT. Con este motivo existen en marcha numerosos estudios intergrupos. CALGB ha finalizado un estudio fase II randomizado de dos ciclos de QT de inducción seguidos de dos ciclos de las mismas drogas en concurrencia con RT. Los tres brazos de tratamiento incluyeron cuatro ciclos de cisplatino $\left(80 \mathrm{mg} / \mathrm{m}^{2}\right)$ combinado con gemcitabine, paclitaxel o vinorelbine. La RT se administró con los dos últimos ciclos de QT hasta una dosis total de $66 \mathrm{~Gy}$. La tasa de respuestas fue similar y la supervivencia media para todos los pacientes fue de 17 meses. sin que exista un claro brazo de tratamiento mejor ${ }^{19}$.

El CALGB actualmente está llevando a cabo un estudio fase III comparando QT de inducción seguida de quimiorradioterapia concurrente vs quimiorradioterapia concurrente sola, usando carboplatino/paclitaxel en ambos brazos.

En esta misma línea, el Grupo Español de Cáncer de Pulmón (GECP), protocolo 00-08, tiene en marcha un estudio con pacientes irresecables estadio IIIA Y IIIB siendo randomizados a QT-RT concurrente y QT posterior, o QT de inducción seguido de QT-RT concurrente, que en la actualidad sigue reclutando enfermos.

\section{Bibliografía}

1. Eberhardt, W. Preoperative chemotherapy followed by concurrent chemoradiation therapy base don hyperfractionated accelerated radiotherapy and definite surgery in locally advanced non-small-cell luna cancer: mature results of a phase II trial. J Clin Oncol 1998; 16:622-34. 
2. Strauss, GM. Neoadyuvant chemotherapy and radiotherapy followed by surgery in stage IIIA non-small-cell carcinoma of the lung: report of a Cancer and Leukemia Group B phase II study.J Clin Oncol 1992; 10:1237-44.

3. Albain, KS. Concurrent cisplatin/etoposide plus chest radiotherapy followed by surgery for stages IIIA (N2) and IIIB nonsmall-cell lung cancer: mature results of Southwest Oncology Group phase II study 8805. J Clin Oncol 1995; 13: 188092.

4. Albain, KS. Proc Am Soc Clin Oncol 1999; 18:467a (abstr 1801).

5. Grunenwald, DH. Benefit of surgery after chemotherapy in stage IIIB (T4 and/or N3) non-small-cell lung cancer. J Thorac Cardiovasc Surg 2001; 122:796-802.

6. Choi, NC. Potencial impact on survival of improved tumor downstaging and resection rate by preoperative twice-daily radiation and concurrent chemotherapy in stage IIIA non-smallcell lung cancer. J Clin Oncol 1997; 15:712-22.

7. Eberhardt, W. Preoperative chemotherapy follwed by concurrent chemoradiation therapy based on hyperfractionated accelerated radiotherapy and definitive surgery in locally advanced non-small-cell lung cancer: mature results of a phase II trial. J Clin Oncol 1998; 16:622-34.

8. Thomas, M. Impact of preoperative bimodality induction twicedaily radiation on tumor regression and survival in stage III non-small-cell lung cancer.J Clin Oncol 1999; 17: 1185-93.

9. Meko, J. Neoadyuvant therapy and surgical resection for locally advanced non-small-cell lung cancer. Semin Radiat Oncol 2000; 10:324-32.

10. Dillman, RO. A randomized trial of induction chemotherapy plus high-dose radiation versus alone in stage III non-small-cell lung cancer. N Engl J Med 1990; 323:940-5.

11. Le Chevalier, T. Radiotherapy alone versus combined chemotherapy and radiotherapy in nonresectable non-small-cell lung cancer: first analysis of a randimized trial in 353 patients. J Natl Cancer Inst 1991; 83:417-23.

12. Sause, WT. Radiation Therapy Oncology Group (RTOG) 88-08 and Eastern Cooperative Oncology Group (ECOG) 4588: preliminary results of a phase III trial in regionally advanced, unresectable non-small-cell lungcancer. J Natl Cancer Inst 1995; 87:198-205.

13. Dillman, RO. Improved survival in stage III non-small-cell lung cancer: seven-year follow-up of cancer and leukaemia group $B$ (CALBG) 8433 trial. J Natl Cancer Inst 1996; 88: 1210-15.

14. Schaake-Konning, C. Effects of concomitant cisplatin oral radiotherapy on inoperable non-small-cell lung cancer. $N$ Engl J Med 1992; 326: 524-30.

15. Clamon, G. Radiosensitization with carboplatin for patients with unresectable stage III non-small-cell lung cancer: a phase III trial of the Cancer and Leukemia Group B and the Eastern Cooparetive Oncology Group. J Clin Oncol 1999; 17:4-11.

16. Lee, JS. Concurrent chemoradiation therapy with oral etoposide and cisplatin for locally advanced inoperable non-small-cell lung cancer: radiation therapy oncology group protocol 91-06. J Clin Oncol 1996; 14:1055-64.

17. Furuse, K. Phase III study of concurrent versus sequential thoracic radiotherapy in combination with mitomycin, vindesine, and cisplatin in unresectable stage III non-small-cell lung cancer. J Clin Oncol 1999; 17: 2692-9.

18. Curran, WJ. Phase III comparison of sequential versus concurrent chemoradiation for patients with unresected stage III nonsmall-cell lung cancer (NSCLC): initial report of radiation therapy oncology group.RTOG 9410. Proc Am Soc Clin Oncol 2000; 19:484 (abstr 1891).

19. Vokes, EE. Randomized phase II study of cisplatin with gemcitabine or paclitaxel or vinorelbine as induction chemotherapy followed by concomitant chemoradiotherapy for stage IIIB nonsmall-cell lung cancer. J Clin Oncol 2002; 20: 4191-8. 